\title{
Building a More Robust Qualitative Methodology in the Study of Digital Communities in Virtual Worlds: Revisiting the Case Study Approach
}

\author{
Donna Z. Davis \\ University of Oregon
}

This paper addresses methodological challenges of research in the increasingly complex arena of virtual worlds and virtual reality. Case studies can be a valuable research tool, yet traditional approaches do not take into consideration the nuances of virtual experiences. Addressing what appears to be a growing body of evidence that points to a lack of rigor in research methodology in this field, specifically as evidenced by inconsistent research methods and analysis, this paper proposes an adaptation of the traditional multimethod qualitative case study method to address the affordances of virtual worlds and VR. It extends Yin's (2009) traditional sources of evidence necessary to create a robust case study to virtual environments including digital/virtual forms of: documents, archival records, interviews, direct observation, participantobservation, and physical artifacts.

Keywords: qualitative research, case study, virtual worlds, virtual reality, research methods

\section{INTRODUCTION}

Although virtual reality (VR) technology has been around for nearly 200 years, since Sir Charles Wheatstone developed binocular vision and ultimately the stereoscope in the mid-1800s, followed by Morton Heilig's Sensorama about 100 years later, the technology as it is defined and researched today is still in an evolutionary and nascent stage. VR headsets offer experiences that completely immerse an individual in a multisensory and embodied way in the platform. The commercial, industrial, military, gaming, social, healthcare, and educational content for VR headsets continue to gain in popularity with the introduction of contemporary VR headsets such as the HTC Vive and the Oculus Rift, S, and Quest. Yet, mass adoption has still struggled (Petty, 2018; Fogel, 2019; Jenkins, 2019). Most of that struggle is attributed to issues such as poor user experience, lack of quality (or enough) content, a lack of understanding and/or a reluctance to embrace the technology, and cost (Perkins Cole, 2020).

In the spring of 2020, the emergence of COVID-19 forced people across the globe to shift to remote work and socialization, offering a new urgency to innovate VR platforms in a way that would drop these barriers to adoption. In July 2020 Facebook Research revealed a prototype of an entire redesign of VR headsets that look more like sunglasses (Facebook Research, 2020) and Apple is likewise developing the N301 headset that includes a "mostly fabric body" (Porter, 2020). Still, these headsets are likely still years away. New players have also entered the VR landscape in their quest to tap into the virtual event and education arenas such as the Wookey Project Corporation's Sansar, Learnbrite - who positions themselves as the "World's First HTML5 VR E-Learning Platform (Learnbrite, 2020). As the crowds rush in, much 
research will continue to help both industry and the academy better understand both the power and the impotence of these technologies as they continue to reach mass adoption.

However, beyond the importance and relevance of understanding the individual media effects, entertainment value, pedagogical impact, efficacy in training and healthcare, and sales data of virtual environments and experiences, increasingly it will be important to understand the evolution and efficacy of entire communities and digital cultures that form in these immersive environments, potentially even more so in an era of medically mandated social distancing. It's even more important to understand the strengths and weaknesses of these emerging cultures, especially when their digital nature also creates opportunity for international and intercultural interaction, communication, and connection. To that end, much can be learned from decades of research of screen-based virtual worlds, such as those in the game space and social worlds such as Second Life.

\section{A Problem With Rigor}

Although much research has been done in these environments, the methodological approaches, much like the technology itself, is still evolving, both in VR and in immersive screen-based virtual worlds.

Take, for example, a recent systematic analysis research of immersive virtual reality applications for educational uses (Randianti, Majchrzak, Fromm, \& Wohlgenannt, 2020). The study found that the most commonly applied quantitative analytical method among the studies was the t-test. Interestingly, the authors reported, "it was a bit counterintuitive that most papers had no explicit or recognizable data analysis method." Only four of the papers utilized qualitative approaches and among those, only observation and focus group discussions were reported.

Similarly, a meta-scientific analysis of the statistical power reported results, and the validity of research specific to the psychology of virtual reality and immersive environments (Lanier, et al., 2019) found a "high incidence of errors in statistical reporting, and a general lack of transparency with respect to the availability of the study data." This issue was further amplified in yet another study that found "the state of efficacy and effectiveness studies of VR interventions still remains weak with some applications due to smaller sample sizes, lack of randomized control trials, and a gap in reporting key intervention qualities, dosage, and outcomes" (Trahan, Smith, \& Talbot, 2019).

These meta-analyses each point to an issue with methodological rigor in the current state of research in VR and immersive environments. Perhaps even more perplexing is that overwhelmingly these studies reference quantitative research methods. This raises an important question about data as it is collected in virtual environments. These platforms arguably live between traditional text, image, and video-based social and broadcast media and the digital parallel to the physical world where data and behavior may more likely portray the attributes of the physical world. To better understand these nuances, qualitative research may also provide insight into understanding the way data is perceived, collected, analyzed, and reported.

Boellstorff, Nardi, Pearce, and Taylor (2012) addressed the ethnographic approach to studying virtual worlds with a specific focus on research design and preparation, participant observation, conducting interviews, and data collection from chat logs, screenshots, and other digital attributes of the virtual world. This paper proposes to extend that work to address emerging issues in immersive embodied cultures that form, function, and dissolve in the rapidly changing landscape of communities in both virtual worlds and VR.

In qualitative communication research, case studies have long been utilized as a method of collecting, organizing, and reporting descriptive data. The strength of case analysis is in the development of an indepth description and analysis of events, programs, activities, and organizations (Creswell \& Poth, 2017). Building on Yin's (2009) principles of collecting case study evidence, we propose a model that addresses the contextualization of that evidence when it collected in virtual environments that are traditionally defined by the common features of presence, interactivity, synchronicity, individuals represented as avatars, and facilitated by a network of computers (Mbryonic.com, 2019) (or increasingly in the cloud). The recommendations emerge from more than a decade of ethnographic research of social virtual communities in immersive environments, both in virtual worlds and in VR. 


\section{THE CASE STUDY APPROACH}

As previously indicated, scholarly research has traditionally and overwhelmingly employed experimental methods to understand behaviors in virtual worlds. This approach is helpful to dissect the psychological effects of engaging in virtual worlds. However, VR, social virtual worlds and game cultures have become embedded in popular culture to the extent that the platforms themselves, as well as organizations and brands, are exploring their potential uses (NewsCred, 2018; American Cancer Society, 2020). Although brands continue to find limited success in what some may call a legacy platform such as SL (Barnes, Mattsson, \& Hartley, 2015) other organizations such as the American Cancer Society continue to build social, cultural, and fiscal capital in the environment, raising more than $\$ 250,000$ annually from avatars in the virtual world. What can they and other communities teach us about functioning in virtual worlds, especially as the online game, Fortnite drew nearly 11 million people into the game simultaneously for a concert in 2019 (Webster, 2019). A qualitative approach would provide the insights that explain these phenomena that quantitative metrics cannot.

Lindlof (1995) argued that qualitative methods are necessary for the early stages of a cultural phenomenon when little is understood about the systems, functions, and beliefs unique to that environment. Through qualitative exploration, the development of a rich, descriptive narrative can lead to quantifiable themes, norms, and standards used for further study. And although VR and virtual worlds have been studied for decades, the technologies and their uses are changing so rapidly that what may be appropriate today is potentially obsolete within months. Consider, for example, the networked gaming culture that has evolved from the early MMORPGs that no longer exist (Altay, 2009; Davenport \& Fenlon, 2019; Olivetti, 2012) to the contemporary blockbuster successes of both Minecraft and Fornite, to the highly immersive and graphicly rich and realistic experiences in a VR headset. The technological attributes of each of these platforms differ dramatically, as does the audience typology in an era of digital natives.

The rigor of qualitative research is also more robust when triangulating data collected through the use of multiple methods. To that end, Yin (2009) proposed six sources of evidence for an effective case study protocol including documents, archival records, interviews, direct observation, participant-observation, and physical artifacts. Although not all sources will be relevant for all cases, effective case study analysis should include as many of these sources as possible. Although each of these sources of evidence is worthy of its own explication, this overview provides an introduction to understanding the implications of "virtual evidence" that can be far more organic than physical evidence (See Figure 1). In many regards, this paper serves as such a case study of the social virtual world Second Life (SL). In addition to a review of current literature, the examples provided result of nearly fourteen years of the author's ethnographic study in the platform.

SL is one of the largest, oldest, and most stable of the existing social virtual worlds, with reportedly more than 800,000 active accounts before the COVID-19 pandemic (Second Life, 2002) and having just celebrated its $17^{\text {th }}$ year in existence. Recent reports suggest the population surged to nearly a million since the beginning of the pandemic (Dodds, 2020).

Note: for clarification, in studies of virtual worlds, it is common to refer to the virtual world and the "real" world. We argue that there are a virtual world and a physical world where virtual environments are inhabited by real humans operating via their avatar and that individuals have real relationships and real "experiences" in these digital environments. Although they may be digitally enhanced or manipulated, the physical world experiences similar manipulations making "real" "virtual" and vice versa. 
FIGURE 1

TRADITIONAL CASE STUDY EVIDENCE VS DIGITAL EVIDENCE

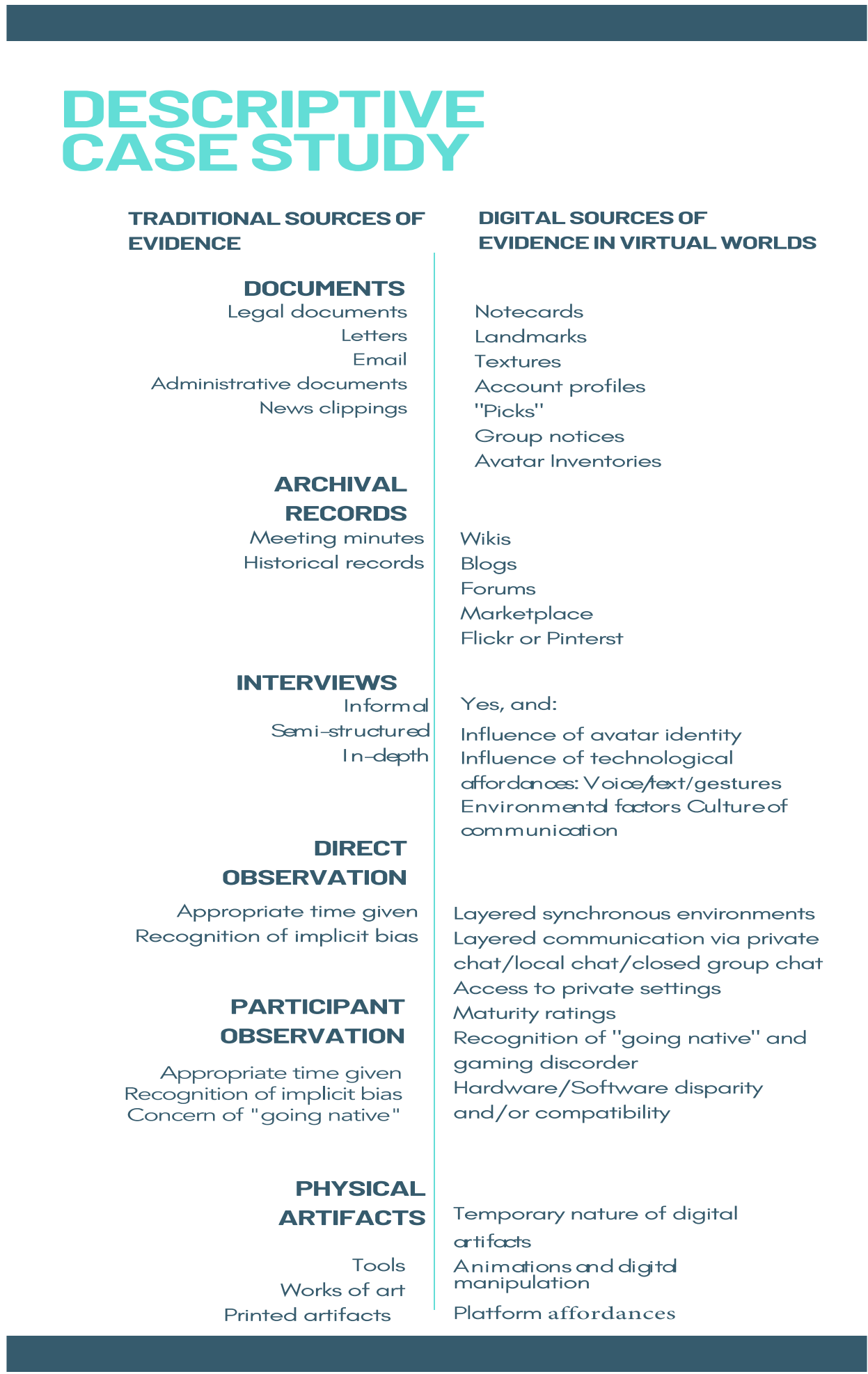




\section{Documentation}

Documentation in the physical world includes a variety of possibilities such as letters, email correspondence, diaries, calendars, agendas, meeting minutes, administrative documents, news clippings, and more. What is a document in a virtual environment? Using the social virtual world Second Life (SL) as an example, documents can take many forms (Davis \& Moscato, 2018). Nearly each of the aforementioned documents exist in the SL platform both "in-world" and beyond the platform. The program allows users, or "residents" to create shareable content. For instance, "notecards" are a digital blank page that can be used for diaries, letters, minutes, and the equivalent of email correspondence. However, there are also character limits in these digital documents, as well as limits on the ability to create graphics. These notecards are basic text-only with exception of the ability to link objects within the notecard - such as including a clickable "landmark" that will teleport the avatar to a desired location.

"Textures" (or graphic images) can also be linked in the notecard to open independently. These textures are additional documents that are equally interesting as searchable and analyzable objects. The qualitative researcher would need to understand how these documents are shared, including their privacy settings. For example, images can be uploaded either as taken in the virtual world or as uploaded from a hard drive and saved as textures. These textures can then be used by the texture owner or shared with others. Privacy features are also built into the platform to allow the content creator to allow or restrict the next "owner" (if they have shared it) to either copy or transfer the object to others. Savvy users also know that by sharing an image, anyone can also create a screen capture of an image that is not meant to be copied or shared. These images can also be exported to a computer hard drive and shared with other social media. In fact, there is a very active Facebook group of more than 41,000 members (Facebook, Second Life Friends, 2020). This cross-platform communication represents the bridge between the virtual world and the physical world, yet on another social media platform. Members may be represented as their avatars or via their physical world identities. This evidence also points to interesting questions about identity and the links between human and avatar as well as across digital destinations.

Another source of evidence of interest to the virtual world researcher is in both individual and group documents in the forms of profiles and group information. Avatar profiles contain information including how long they've been in SL, whether they have payment information attached to their accounts, what groups they belong to, an "about" section where they can choose to say something about themselves (or others). Profiles also include tabs linked to the Web; what "Interests" they may have; "Picks" that include images, landmarks, and information about their favorite people, places, or activities; a "Classified" tab if they sell a product or service; and a link to their " $1{ }^{\text {st }}$ Life," often including a photo of the human account holder. A final tab offers the user a page to leave themselves notes about the friend whose profile they are looking at. As a researcher, the notes tab can be very helpful for abbreviated but critical field notes about the individual. Profiles are rich in information including the use of images as well as the choice of content they include or make private. Some avatars refer to themselves as "profile surfers" as they make assumptions and decisions about individuals based on their profiles without ever needing to meet or speak with the individual.

Similarly, group documents are very informative. Groups can be created for any number of people of two or more. Although global statistics on groups are not available, there are groups of more than 40,000 people such as the Omega Applier Systems support group. The Omega Applier System is a popular accessory for personalizing avatars with customized skins, makeup, hair, etc. Likewise, publicly available group information may include information about the group founder, what the group's purpose is, how many members they have and who those members are, and links to experiences they may have built or host, and group notices. The "notices" feature allows groups to send notices to their members (announcements, alerts, advertisements, and such), alerting them about upcoming events, sales, or other relevant information for group members. Of important note, group notices have a character limit and will only be archived for 14 days. As such, these documents will not serve as archival records unless they are captured and saved within the 14-day limit.

Another important document source is in every avatar's "Inventory." Inventories include nineteen default files including animations, calling cards, clothing, gestures, landmarks, notecards, objects, photos, 
sounds, textures, and more. Each file can hold multiple "elements." Some avatars, especially those who have been in SL for many years may have tens of thousands of items in their inventories. An inventory of inventories can be very revealing about the activities and evolution of an avatar's time in the virtual world. These inventories are private and as such any documentation from inventories would have to be captured and requested (Figure 2).

\section{FIGURE 2 SL INVENTORY SCREEN CAPTURE}

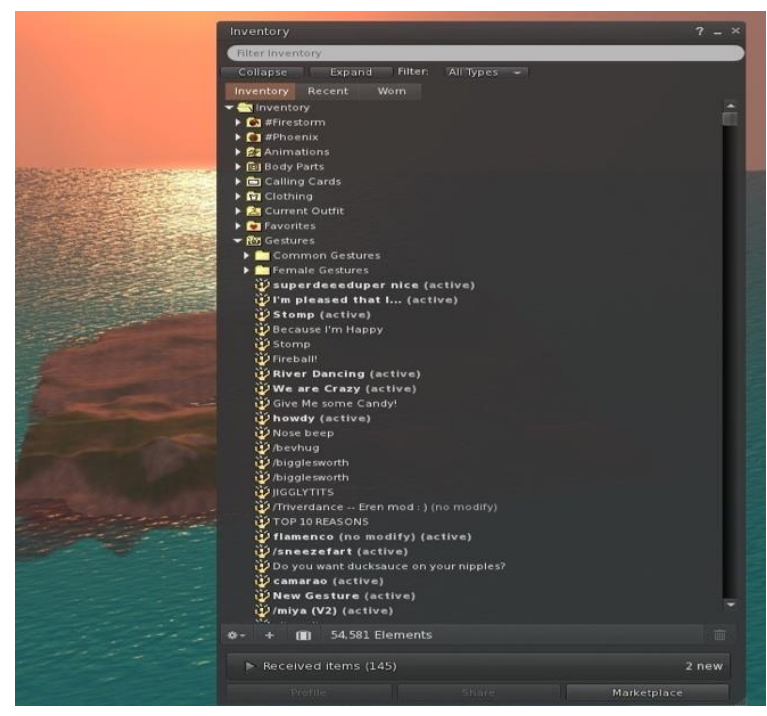

\section{Archival Records}

Archival records in online social games and virtual worlds can be difficult to attain. Much of the statistical data such as daily logins, current total numbers of accounts, and such is proprietary and accordingly may not be available. However, multiple commercial media provide the available data. Similarly, community wikis and blogs are often excellent sources of archival records. For example, the SL General Discussion Forum (Second Life Forum, nd.) archives date back to 2011. Another excellent source of evidence can be found in discussion group archives. Not all groups archive their discussions (and should report that they do, if they are publishing their archives, as it may violate the SL Terms of Service due to privacy concerns). Additional sources of archival records can be find at sources such as the SL Marketplace (https://marketplace.secondlife.com/) to search goods for purchase as well as on photo sharing website, Flickr (https://www.flickr.com/groups/_traveler/).

Additional archival records can be collected from organizations that host activity in SL. For example, the American Cancer Society hosts an annual Relay for Life of Second Life (American Cancer Society, 2020). As evidenced on the RFL of SL website, archival data available include calendars, region information, event announcements, historical information, organizational information, fundraising guidelines, and a current "scoreboard" that keeps track of the top events, individuals, and companies" fundraising statistics. In addition to collecting documents from sources in-world, the use of archival records from the organization's website (when websites, wikis, blogs, and social media pages are available) can help begin to triangulate data in a way that creates a more complete picture of the case example.

\section{Interviews}

At the core of ethnographic method are observation and interviews. Many studies have utilized these methods in virtual world research, given the robust communities and vibrant history of the platform (Davis, 2013; Martey \& Consalvo, 2011). In a virtual world, the same best practices of collecting data through interviews still apply. Interviews can be informal, in-depth, and/or semi-structured. Recruitment requires 
building trust and rapport, and seeking permissions, however, when interviewing avatars, consider how digital affordances may influence the context of the interview. Consider, for example, interviewing a female avatar who may be a male human. What questions might be asked, for what purpose, and to what affect? How and why would gender be relevant both in the physical and virtual worlds? Do we accept the data for the value it brings at its face? How might this be different than our expectation of truth from face-to-face interviews?

Additionally, conducting interviews with avatars is still widely misunderstood. Some individuals (and previously university Institutional Review Boards) may argue that avatars aren't actually people and as such don't need to fall under the same rigor of protections - even though avatars only function via the actions of their human operator (Fairfiled, 2012; Zimmerman \& Chapman, 2020). Others might argue that interviews with digital interlocuters can't be trusted as there is no way to verify the data and that deception may be easier without face-to-face engagement. However, the same logic would similarly invalidate interviews conducted in telephone research or even in the increasingly popular Mechanical Turk (MTurk).

Finally, conducting interviews in virtual worlds is accompanied by digital data and affordances that make data collection potentially both challenging and/or more efficient. Although many virtual worlds offer both voice and text communication, the majority of communication is conducted via text. Therefore, interview transcripts are digitally available upon completion of the interview. Spending adequate time in the virtual world to understand the "language" of the environment (Table 1) (Davis, 2013) is critical to effective interpretation of the interview data. Text abbreviation and misspelling is very common in text chat.

It is still important to determine the most appropriate interview form. Some individuals are more comfortable in voice (consider a blind participant) and should be given that option. Voice recording is also available in most virtual worlds, as it is also a standard feature now in VR.

Interviews conducted in a virtual environment can also create environmental factors that should be taken into consideration. As remote meetings are teaching us now, the computer-mediated background can influence the interaction between the interviewer and the participant. Finding a safe, comfortable, and private location is important. Establishing each of these elements (location, text, voice, privacy) must be done prior to the interview.

TABLE 1

COMMON ABBREVIATIONS USED IN SECOND LIFE

\begin{tabular}{ll}
\hline Abbreviation & Meaning \\
\hline$;$ or $:$ & Emoticon that may also appear as : ) or : ( to represent a smile or sad face \\
\hline afk & Away from keyboard (or have stepped away from their computer) \\
\hline Alt & alternate account, often people create more than one avatar for different uses \\
\hline AO & Animation override (creates a more natural movement) \\
Av or Avi & abbreviation for avatar \\
Brb & Be right back \\
D/s & Dominant/submissive (to reflect master/slave roles in Gorean or BDSM role \\
& play) \\
FL or fl & First life (an alternative way of saying 'real life') \\
HB or hb & Hurry back \\
HUD & Head-up display ("worn" on an avatar to provide data) \\
idk & I don't know \\
IM & Instant message \\
kk & Okay \\
lag & When the system is loading slowly often causing freezing \\
LL & Linden Lab - creators of Second Life \\
LM & Landmark - a digital address or location in Second Life \\
\hline
\end{tabular}




\begin{tabular}{ll}
\hline LOL & Laugh out loud \\
NC & Notecard - used to save text in a file in inventory \\
Noob or newb & Someone new to the virtual world \\
OMG & Oh my God! (Oh my gosh!) \\
ppl or peeps & People \\
RL or rl & Real life \\
Rofl & Rolling on the floor laughing \\
SL & Second Life \\
SLT & Second Life time (SLT)- Is the same as Pacific time (home of Linden Labs) \\
& and is used as the universal time in Second Life for scheduling purposes \\
SLurl & Second Life URL \\
Smh or smdh & Shake my head or shake my damn head \\
ToS & Terms of Service \\
TP & Teleport \\
wb & Welcome back \\
\hline
\end{tabular}

\section{Direct Observation}

Direct observation is another valuable source of evidence in case study design. Direct observation, even in a virtual world, allows the researcher what should be an impartial view of events in real time and helps create context (Barzell \& Odom, 2008, Guitton, 2012, Guitton, 2015). As with most ethnographic data collection, this method is time consuming and can be misleading without adequate coverage of events to get a complete picture. In working with multiple highly evolved social communities in SL, on multiple occasions journalists would "parachute" into the world to cover a story. The journalists would often expect to find certain behaviors or story lines and the communities felt the resulting coverage reflected predetermined angles and biases, often with dystopic and marginalized themes. Without the deep and meaningful understanding of the underlying stories of the individuals and the communities in which they function, dystopic stereotypes are often reinforced. Researchers who use direct observation without additional evidence through triangulation with mixed methods run the same risk as these storytellers.

Additionally, because the virtual world offers complex layers of multisensory information, observation can be manipulated in interesting ways. Returning to the Relay for Life example, the majority of the funds raised in SL are generated through events (Davis \& Moscato, 2018). Should a researcher log in to the virtual world expecting to observe an event, they may attend a very crowded concert event and discover no one speaking. Although texting openly with others in a public chat window is very common at events, in some cases, the individuals attending the event may also be engaged in private chats, closed group chats, or be away from their keyboard (afk). These activities cannot be seen by the casual observer (like walking into a room where you cannot hear any conversations while everyone is busy text chatting on their cellphones). Although these examples are common in the virtual world, they may also teach us about an increasingly connected population and the way they behave both online and offline.

Similarly, logging in to a popular venue with high traffic numbers without having an event schedule, the researcher may find an empty venue. Just as concert venues are empty between events, logging into an empty venue without context can be misleading.

Likewise, virtual environments have limitations on how much data they can manage at any given time. In a recent popular event in AltSpaceVR, the location filled to capacity quickly. For individuals who logged in on time (but later than those who filled the room early), they were sent to an identical environment, or second room, that was live streaming the video from the main event space. A casual observer may not realize that their more sparsely filled environment was in fact an overflow space, or as in this case one of several overflow spaces, also potentially misleading the observer about the popularity of the event.

Another possible confusion the casual observer may experience are regions in virtual worlds that may have maturity ratings that limit access to individuals. If a casual observer is not aware of these settings, they may limit access to General $(G)$ population areas only where most activities are located in Mature (M) or Adult (A) rated regions. Without some time and understanding, they may also limit access to adult regions 
for fear they are pornographic or gratuitously violent. This is often not the case, rather, land has been purchased by individuals who may not have control over the settings but have no intention of hosting "adult" content.

\section{Participant Observation}

In order to overcome many of the shortcomings of the direct observation method of evidence collection, participant observation allows the researcher the opportunity to become embedded in a community and observe as a member of that community. As part of the community, the researcher is allowed to access events, activities, groups and individuals more intimately. As with direct observation, this method is very time consuming, yet it offers more meaningful insight into the interpersonal behaviors and motivations of the community members. As with its analog cousin, the risks involved with this method is the increased potential bias, "going native," or becoming so involved that the time consumption becomes overwhelming. This may have an exaggerated effect in virtual environments because of the attributes of these spaces that are considered addictive. After years of controversy, in 2019, the World Health Organization officially recognized "gaming disorder" as a mental health condition (Rettner, 2019). Participants in the social communities in SL who have participated in ongoing studies of the effects of engagement in virtual worlds have occasionally reported a sense of "addiction" and certainly experience "flow states" (Faiola \& Smyslova, 2009). A participant observer would need to be aware of their own predisposition to becoming too close to their study participants and to the potential allure of spending time in the world in the name of research.

\section{Physical Artifacts}

The final source of evidence as recommended by Yin in the development of effective case study design is the collection of physical artifacts. This method is particularly interesting in its interpretation of digital artifacts. Yin suggests this method would collect artifacts such as tools, works of art, or printed artifacts of digital objects. These objects should offer insight into the culture and operations of the community, event, or organization.

In their study of people with Parkinson's disease, Davis and Boellstorff (2016) articulated the notion of digital objectified states, recognizing "the importance of digital materiality' among their community members. These digital artifacts, including elaborate architectural builds, clothing designs created for historical role playing in the virtual world, and virtual homes, for example, were incredibly important to the individuals in this community. Much of that value was a result of ownership of the fruits of their creativity and the accompanying sense of purpose that those creations provided these participants. And yet, in the case of architectural builds, these creations were also very temporary. In the virtual world, regions and lands are often deleted or sold and reconfigured with great frequency. Just as the video games that come and go, although the platform has remained stable for nearly two decades, the lands of SL are built and maintained by the members of its community. If those residents lose interest, can no longer afford their land, or have changing life circumstances that pull them away, their creations go with them. In that regard, the virtual world is quite different from the physical world in that virtual art museums are much less likely to survive than a well-endowed museum or gallery.

For a brief period, Linden Lab, the founding organization that has hosted SL for the past 17 years created a Linden Endowment for the Arts (LEA) to showcase the creative artistry of the many residents in SL. Linden Lab proclaimed, "The Linden Endowment of the Arts is an official Linden Community Partnership program whose purpose is to help new artists, cultivate art in SL, and foster creativity, innovation, and collaboration within the art community" [32]. Many of the extraordinary builds were in place for only brief amounts of time and now only exist in resident inventories. Contrarily, Canadian artist Bryn Oh has used SL as a creative platform and has archived her work in blogs since 2008 (Bryn Oh, 2020), on YouTube, now with 79 videos and nearly 2,000 subscribers, and in physical artifacts of her work. This artist represents another case where each of the six sources of evidenced for an effective case study, both virtually in SL and across other mediated social platforms, could easily be utilized to reflect the similarities and differences of virtual and physical data. Unfortunately, she is the exception to the rule, especially as 
we consider artifacts as evidence. Digital artifacts in SL are often only archived in the memories of those who visited or experienced them in the time they were engaged in-world. As such, it is important to remember the importance of creating images through screen shots, video and audio recording of events, and archiving chat logs as reliable and sustainable evidence.

\section{Extension of Physical Evidence}

As discussed, effective case study analysis suggests utilization of documentation, archival records, interviews, direct observation, participant observation and physical artifacts. Yin (2009) also suggests that these six sources of evidence are most effective when three principles are also applied:

- Use multiple sources of evidence

- Create a case study database

- Maintain a chain of evidence

Many of the attributes of virtual worlds and VR have been reviewed in the discussion of how to consider their influence on the multiple sources of evidence. However, additional questions must also be considered when navigating these approaches. For example, when collecting data about virtual worlds and VR, what role does interactivity play? In many VR games, interactivity occurs with the environment but not with other individuals.

That in mind, also consider the role of the platform software and hardware. For instance, in a study of people with disabilities in virtual worlds, the researchers had intended to provide VR headsets to the individuals participating in SL. However, when the headsets were finally made available for consumer use, the cost - not only of the headset, but of the computers required to run the programs - made it cost prohibitive. Additionally, several of the people in the community had no interest in wearing a headset for numerous reasons such as comfort, weight, fear of claustrophobia, reports of and being prone to motion sickness, and a general lack of interest. Hardware and software become artifacts that need to be addressed as they relate to the experience of populations studied. Researchers in these technological spaces must consider how the affordances (or lack thereof) of virtual worlds and VR can change the human experience.

\section{FUTURE CONSIDERATIONS}

In approaching a case study design, each of the forms of evidence collection offer strong merit. Especially when considering the influence of the hardware and software on the experience of the user, other accessibility issues, including cost, must be addressed and resolved in order to identify potential issues in equity and inclusion. Ultimately, key features such as built-in gestures, emoticons designed to help users express emotions, available animations, and the ability to customize avatar identity might influence the way people experience these environments. Just as text abbreviations become a form of language, features that create movement and emotional expression are digital artifacts that extend beyond the traditional physical experience.

We return to the original question posed: why do organizations want to create experiences, events, and communities in virtual worlds and VR? Case study analysis may be the best method to answer that question when carefully articulating what the platforms are capable of, what they are not capable of, and how users may choose to (or not chose to) use them. They represent exciting potential environments in our newly prioritized virtually connected world. Understanding their best use will take thorough review from multiple perspectives.

\section{REFERENCES}

Altay, O. (2009). The MMO graveyard - Dead MMORPGs. MMOhuts.com. Retrieved from https://mmohuts.com/news/the-mmo-graveyard-dead-mmorpgs/

American Cancer Society. (2020). Relay for Life of Second Life. Retrieved from https://secure.acsevents.org/site/STR?pg=entry\&fr_id=97294 
Barnes, S., Mattsson, J., \& Hartley, N. (2015, March). Assessing the value of real-life brands in virtual worlds. Technological Forecasting and Social Change, 92, 12-24. ISSN 0040-1625. https://doi.org/10.1016/j.techfore.2014.10.017

Bell, M.W. (2008). Towards a definition of "virtual worlds". Journal of Virtual Worlds Research, 1(1). Retrieved January 24, 2009, from http://journals.tdl.org/jvwr/article/view/283/237

Boellstorff, T., Nardi, B., Pearce, C., \& Taylor, T.L. (2012). Ethnography and Virtual Worlds: A Handbook of Method. Princeton, NJ: Princeton University Press.

Creswell, J., \& Poth, C. (2017). Qualitative Inquiry and Research Design (Fourth edition). Sage, Thousand Oaks, CA.

Davenport, J., \& Fenlon, W. (2019, May 21). 25 MMOs that lived and died since World of Warcraft launched. PC Gamer. Retrieved from: https://www.pcgamer.com/mmo-graveyard/

Davis, D. (2013). A study of relationships in online virtual environments: Making a case for conducting semi-structured interviews with avatars and what we can learn about their human operators. In N. Sappleton (Ed.), Advancing Social and Business Research Methods with New Media. Hershey, PA: IGI Global.

Davis, D., \& Boellstorff, T. (2016). Compulsive Creativity: Virtual worlds, disability, and digital capital, International Journal of Communication, 10, 2096-2118. Retrieved from http://ijoc.org/index.php/ijoc/article/view/5099/1639

Davis, D., \& Moscato, D. (2018) The Philanthropic Avatar: An Analysis of Fundraising in Virtual Worlds Through the Lens of Social Capital. International Journal of Strategic Communication, 12(3), 269-287. DOI: 10.1080/1553118X.2018.1464007

Dodds, L. (2020, March 26). Second Life enjoys a surprising renaissance as social distancers flock to virtual worlds. The Telegraph. Retrieved from https://www.telegraph.co.uk/technology/ 2020/03/26/second-life-enjoys-surprising-renaissance-social-distancers/

Facebook Research. (2020). Holographic optics for thin and lightweight virtual reality. Retrieved June 29, 2020, from https://research.fb.com/blog/2020/06/holographic-optics-for-thin-and-lightweightvirtual-reality/

Facebook, Second Life Friends group. (2020). Retrieved from https://www.facebook.com/groups/155844374435030

Faiola, A., \& Smyslova, O. (2009). Flow Experience in Second Life: The Impact of Telepresence on Human-Computer Interaction. In A.A. Ozok, P. Zaphiris (Eds.), Online Communities and Social Computing. OCSC 2009. Lecture Notes in Computer Science (vol. 5621). Springer, Berlin, Heidelberg.

Fairfield, J.A. (2012). Avatar Experimentation: Human Subjects Research in Virtual Worlds. U.C. Irvine Law Review, 2, 695-772.

Fogel, S. (2019). VR developers say audience adoption is their biggest challenge. Variety. Retrieved April 25, 2019, from https://variety.com/2019/gaming/news/htc-vive-vr-developer-survey-1203197302/ forums/forum/321-general-discussion-forum/page/528/

Guitton, M.J. (2012). The immersive impact of meta-media in a virtual world. Computers in Human Behavior, 28(2012), 450-455.

Guitton, M.J. (2015)._Swimming with mermaids: Communication and social density in the Second Life merfolk community. Computers in Human Behavior, 48(2015), 226-235.

Jenkins, A. (2019). The fall and rise of VR: The struggle to make virtual reality get real. Fortune. Retrieved June 20, 2019, from https://fortune.com/longform/virtual-reality-struggle-hope-vr/

Lanier, M., Waddell, T.F., Elson, M., Tamul, D.J., Ivory, J.D., \& Przybylski, A. (2019). Virtual reality check: Statistical power, reported results, and the validity of research on the psychology of virtual reality and immersive environments. Computers in Human Behavior, 100(2019), 70-78. ISSN 0747-5632. https://doi.org/10.1016/j.chb.2019.06.015

Learnbrite. (2020). Retrieved from https://learnbrite.com/home/

Linden Endowment for the Arts. (2019). Retrieved from lindenarts.blogspot.com

Lindlof, T. (1995). Qualitative communication research methods. Thousand Oaks, CA: Sage. 
Martey, R.M., \& Consalvo, M. (2011). Performing the Looking-Glass Self: Avatar Appearance and Group Identity in Second Life. Popular Communication, 9(3), 165-180. https://doi.org/10.1080/15405702.2011.583830

Mbryonic.com. (2019). 10 best uses of virtual reality VR marketing, 2019. Retrieved from https://mbryonic.com/best-vr-marketing/

NewsCred. (2018). 5 fantastic examples of brands that use virtual reality (VR). Retrieved from https://insights.samsung.com/2018/05/09/5-fantastic-examples-of-brands-that-use-virtual-reality$\mathrm{vr} /$

Oh, B. (2020a). Retrieved from http://brynoh.blogspot.com/

Oh, B. (2020b). Retrieved from https://www.youtube.com/user/BrynOh

Olivetti, J. (2012, May 1). The game archaeologist: When dead MMOs come back to life. Engadget. Retrieved from https://www.engadget.com/2012/05/01/the-game-archaeologist-when-deadmmos-come-back-to-life/

Perkins Cole @ Statista. (2020). Leading obstacles to mass adoption of virtual reality (VR) technologies according to industry experts in first quarter of 2019, 2020. Retrieved from https://www.statista.com/statistics/1098566/obstacles-to-mass-adoption-of-vr-technologies/

Pettey, C. (2018). Here's how technology product managers should plan for virtual reality and augmented reality adoption in the next few years. Gartner. Retrieved September 6, 2018, from https://www.gartner.com/smarterwithgartner/3-reasons-why-vr-and-ar-are-slow-to-take-off/

Porter, J. (2020). Go read this detailed look at Apple's AR and VR headset development. The Verge. Retrieved June 19, 2020, from https://www.theverge.com/2020/6/19/21296674/apple-vr-arheadset-glasses-external-hub-jony-ive-bloomberg-go-read-this

Radianti, J., Majchrzak, T., Fromm, J., \& Wohlgenannt, I. (2020, April). A systematic review of immersive virtual reality applications for higher education: Design elements, lessons learned, and research agenda. Computers \& Education, 147, 103778. ISSN 0360-1315. https://doi.org/10.1016/j.compedu.2019.103778

Rettner, R. (2019). Video game addiction becomes official mental disorder in controversial decision by WHO. LiveScience. Retrieved May 28, 2019, https://www.livescience.com/65580-video-gameaddiction-mental-health-disorder.html\#: :text=According $\% 20$ to $\% 20$ the $\% 20 \mathrm{WHO} \% 2 \mathrm{C} \% 20$ gaming,in\% 20their\%20family\%20relationships $\% 2 \mathrm{C} \% 20$ social

S. Bardzell, S., \& Odom, W. (2008). The experience of embodied space in virtual worlds. Space and Culture, 11(3), 239-259 saw\%20rapid\%20growth\%20for,\%22between\%20800\%2C000\%20and\%20900\%2C000\%22

Second Life General Discussion Forum. (2020). Retrieved from https://community.secondlife.com/

Second Life, Wikipedia. (nd). Retrieved from https://en.wikipedia.org/wiki/Second_Life\#: :text=It\%20

Trahan, M.H., Smith, K.S., \& Talbot, T.B. (2019). Past, Present, and Future: Editorial on Virtual Reality Applications to Human Services. Journal of Technology in Human Services, 37(1), 1-12. DOI: $10.1080 / 15228835.2019 .1587334$

Webster, A. (2019). Fortnite's Marshmello concert was the game's biggest event ever. The Verge. Retrieved February 21, 2019, from https://www.theverge.com/2019/2/21/18234980/fortnitemarshmello-concert-viewer-numbers

Yin, R.K. (2009). Case study research: Design and methods ( $4^{\text {th }}$ ed.). Sage, Thousand Oaks, CA.

Zimmer, M., \& Chapman, M. (2020). Ethical Review Boards and Pervasive Data Research: Gaps and Opportunities. Paper presented at AoIR 2020: The 21st Annual Conference of the Association of Internet Researchers. Retrieved from https://spir.aoir.org/ojs/index.php/spir/article/download/11369/9983 